\title{
Affective Image Content Analysis: A Comprehensive Survey*
}

\author{
Sicheng Zhao ${ }^{\dagger}$, Guiguang Ding ${ }^{\ddagger}$, Qingming Huang ${ }^{\sharp}$, \\ Tat-Seng Chua $^{\S}$, Björn W. Schuller ${ }^{\diamond}$ and Kurt Keutzer ${ }^{\dagger}$ \\ ${ }^{\dagger}$ Department of Electrical Engineering and Computer Sciences, University of California, Berkeley, USA \\ ${ }^{\ddagger}$ School of Software, Tsinghua University, China \\ ${ }^{\sharp}$ School of Computer and Control Engineering, University of Chinese Academy of Sciences, China \\ ${ }^{\S}$ School of Computing, National University of Singapore, Singapore \\ ${ }^{\diamond}$ Department of Computing, Imperial College London, UK
}

\begin{abstract}
Images can convey rich semantics and induce strong emotions in viewers. Recently, with the explosive growth of visual data, extensive research efforts have been dedicated to affective image content analysis (AICA). In this paper, we review the stateof-the-art methods comprehensively with respect to two main challenges - affective gap and perception subjectivity. We begin with an introduction to the key emotion representation models that have been widely employed in AICA. Available existing datasets for performing evaluation are briefly described. We then summarize and compare the representative approaches on emotion feature extraction, personalized emotion prediction, and emotion distribution learning. Finally, we discuss some future research directions.
\end{abstract}

\section{Introduction}

With the rapid development of photography technology and the wide popularity of social networks, people have become used to sharing their experiences and expressing their opinions online using images and videos together with text. This gives rise to a huge volume of multimedia data, which results in urgent demand of processing and understanding the visual content. Compared to low-level visual appearances, humans could better perceive and understand the high cognitive level and affective level of semantics [Zhao et al., 2014a]. Existing works on image content analysis from the computer vision community mainly focus on understanding the cognitive aspects of images, such as object detection. Recently, a lot of research attention has been paid from the psychology, affective computing and multimedia communities to affective level analysis of image content. As what people feel may directly determine their decision making, affective image content analysis (AICA) is of great importance, which can enable wide applications [Chen et al., 2014], ranging from humancomputer interaction to image retrieval.

\footnotetext{
${ }^{*}$ Corresponding author: Sicheng Zhao (schzhao@gmail.com)
}

Specifically, the task of AICA is often composed of three steps: human annotation, visual feature extraction and learning of mapping between visual features and perceived emotions [Zhao et al., 2017a]. One main challenge for AICA is the affective gap, which can be defined as "the lack of coincidence between the features and the expected affective state in which the user is brought by perceiving the signal" [Hanjalic, 2006]. Recently, various hand-crafted or learning-based features have been designed to bridge this gap. Current AICA methods mainly assign an image with the dominant (average) emotion category (DEC) with the assumption that different viewers react similarly to the same image. This task can be performed as a traditional single-label learning problem.

However, labeling the emotions in ground-truth generation is in fact highly inconsistent. Different viewers may have totally different emotional reactions to the same image, which is caused by many personal and situational factors, such as the cultural background, personality and social context [Peng et al., 2015; Zhao et al., 2016; Yang et al., 2017b]. This phenomenon causes the so-called subjective perception problem, as shown in Figure 1. In such cases, just predicting the DEC is insufficient for this highly subjective variable.

To tackle the subjectivity issue, we can conduct two kinds of AICA tasks [Zhao et al., 2016]: for each viewer, we can predict personalized emotion perceptions; for each image, we can assign multiple emotion labels. For the latter one, we can employ multi-label learning methods, which associates one instance with multiple emotion labels. However, the importance or extent of different labels is in fact unequal. In such cases, emotion distribution learning would make more sense, which aims to learn the degree to which each emotion describes the instance [Yang et al., 2017b].

In this paper, we concentrate on reviewing the state-of-theart methods on AICA and outlining research trends. First, we introduce the widely-used emotion representation models and the available datasets for performing AICA evaluation. Second, we summarize and compare the representative approaches on emotion feature extraction, personalized emotion prediction, and emotion distribution learning, corresponding to the affective gap and perception subjectivity challenges. Finally, we discuss potential research directions to pursue. 


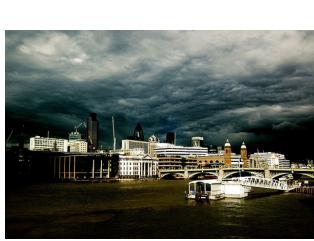

(a) Original image

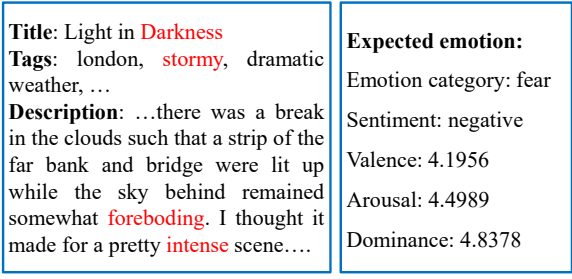

(b) Image metadata

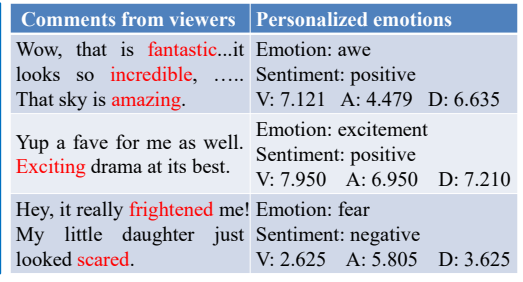

(d) Personalized emotion labels

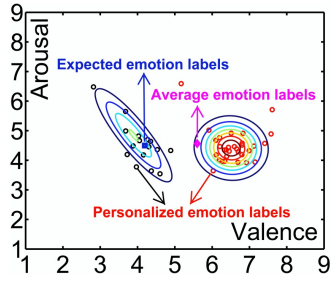

(e) Emotion distribution

Figure 1: Illustration of the image emotion's perception subjectivity phenomenon [Zhao et al., 2017c]. The expected emotions in (c) and the personalized emotions in (d) are assigned using the keywords in red based on the metadata (b) from the uploader and the comments from different viewers. (e) shows the differences of expected, personalized and average emotions, while the contour lines are the estimated continuous emotion distributions by the expectation maximization algorithm based on Gaussian mixture models.

\section{Emotion Models from Cognitive Science Community}

Psychologists mainly employ two typical models to represent emotions: categorical emotion states (CES), and dimensional emotion space (DES). CES models consider emotions to be one of a few basic categories, such as fear and joy, etc. Some typical models include Ekman's six basic emotions [Ekman, 1992] and Mikels's eight emotions [Mikels et al., 2005]. Specifically, emotions can be classified into positive and negative [Zhao et al., 2018b], sometimes including neutral. In such case, "emotion" is usually called "sentiment". DES models usually employ a 3D or 2D Cartesian space to represent emotions, such as valence-arousal-dominance (VAD) [Schlosberg, 1954] and activity-temperature-weight [Lee and Park, 2011]. VAD is the most widely used DES, where valence represents the pleasantness ranging from happy to unhappy, arousal represents the intensity of emotion ranging from excited to calm, while dominance represents the degree of control ranging from controlled to in control. Dominance is difficult to measure and is often omitted, leading to the commonly used two dimensional VA space [Hanjalic, 2006], where $\mathrm{V}$ is easier to recognize in AICA than A.

The relationship between CES and DES and the transformation from one to the other are studied in [Sun et al., 2009]. For example, positive valence relates to a happy state, while negative valence relates to a sad or angry state. CES is easier for users to understand and label, but may not reflect the subtlety and complexity of emotions. DES is more flexible and richer in descriptive power, but absolute continuous values are difficult for users to distinguish and may be unmeaningful because of the lack of agreed-upon standards for subjective emotion rating. CES is mainly used in classification task, while DES is often employed for regression. If discretized into several constant scores, DES can also be used for classification [Lee and Park, 2011]. Ranking based labeling can be applied to ease DEC comprehension difficulties in raters.

Expected emotion is the emotion that the image creator intends to make people feel, while perceived emotion is the actual emotion that is perceived by the image viewers. Discussing the difference or correlation of various emotion models is out of the scope of this paper. We just list some typical emotion models that have been widely used in AICA, as shown in Table 1.

\begin{tabular}{|c|c|c|c|}
\hline Model & Reference & Type & Emotion states/dimensions \\
\hline Ekman & [Ekman, 1992] & CES & $\begin{array}{l}\text { happiness, sadness, anger, disgust, } \\
\text { fear, surprise }\end{array}$ \\
\hline Mikels & [Mikels et al., 2005] & CES & $\begin{array}{l}\text { amusement, anger, awe, contentment, } \\
\text { disgust, excitement, fear, sadness }\end{array}$ \\
\hline Plutchik & [Plutchik, 1980] & CES & $\begin{array}{l}\text { ( } \times 3 \text { scales) anger, anticipation, dis- } \\
\text { gust, joy, sadness, surprise, fear, trust }\end{array}$ \\
\hline Sentiment & & CES & positive, negative, (or neutral) \\
\hline VA(D) & [Schlosberg, 1954] & DES & valence-arousal(-dominance) \\
\hline ATW & [Lee and Park, 2011] & DES & activity-temperature-weight \\
\hline
\end{tabular}

Table 1: Representative emotion models employed in AICA.

\section{Datasets}

The early datasets for AICA mainly come from the psychology community with small-scale images. Recent large-scale datasets are constructed using images from social networks. The released datasets are summarized in Table 2.

The International Affective Picture System (IAPS) is an emotion evoking image set in psychology [Lang et al., 1997]. It consists of 1,182 documentary-style natural color images depicting complex scenes, such as portraits, babies, animals, landscapes, etc. Each image is associated with an empirically derived mean and standard deviation (STD) of VAD ratings in a 9-point rating scale by about 100 college students.

The (IAPSa) dataset includes 246 images [Mikels et al., 2005] selected from IAPS, which are labeled by 20 undergraduate participants with a mean age 19.55 years.

The (Abstract) includes 279 peer rated abstract paintings without contextual content, while the Artistic database (ArtPhoto) consists of 806 artistic photographs from a photo sharing site [Machajdik and Hanbury, 2010], the emotions of which are determined by the artist who uploaded the photo.

The IAPSa, ArtPhoto, and Abstract datasets are categorized into eight discrete categories [Mikels et al., 2005]. Each image is labeled with only one emotion in ArtPhoto, the images in IAPSa may be labeled with more than one emotion, while the detailed votes of all emotions are provided for the images in Abstract, from which one can obtain the DEC and emotion distributions. Totally, 395 emotions are labeled on the 246 images in IAPSa. 228 Abstract images are usually used for emotion classification after DEC selection.

The Geneva affective picture database (GAPED) is composed of 520 negative images, 121 positive images and 89 neutral images [Dan-Glauser and Scherer, 2011], labeled by 60 participants with a mean age of 24 years (ranging from 19 
Proceedings of the Twenty-Seventh International Joint Conference on Artificial Intelligence (IJCAI-18)

\begin{tabular}{|c|c|c|c|c|c|c|c|}
\hline Dataset & Reference & \# Images & Type & \# Annotators & Emotion model & $\mathbf{E} / \mathbf{P}$ & Label detail \\
\hline IAPS & [Lang et al., 1997] & 1,182 & natural & $\approx 100$ (half $\mathrm{f})$ & VAD & $\mathrm{P}$ & empirically derived mean and standard deviation \\
\hline IAPSa & [Mikels et al., 2005] & 246 & natural & $20(10 \mathrm{f}, 10 \mathrm{~m})$ & Mikels & $\mathrm{P}$ & at lease one emotion category for each image \\
\hline Abstract & [Machajdik and Hanbury, 2010] & 279 & abstract & $\approx 230$ & Mikels & $\mathrm{P}$ & the detailed votes of all emotions for each image \\
\hline ArtPhoto & [Machajdik and Hanbury, 2010] & 806 & artistic & - & Mikels & $\mathrm{E}$ & one DEC for each image \\
\hline GAPED & [Dan-Glauser and Scherer, 2011] & 730 & natural & 60 & Sentiment, VA & $\mathrm{P}$ & one DEC and average VA values for each image \\
\hline MART & [Alameda-Pineda et al., 2016] & 500 & abstract & $25(11 \mathrm{f}, 14 \mathrm{~m})$ & Sentiment & $\mathrm{P}$ & one DEC for each image \\
\hline devArt & [Alameda-Pineda et al., 2016] & 500 & abstract & $60(27 f, 33 m)$ & Sentiment & $\mathrm{P}$ & one DEC for each image \\
\hline Tweet & [Borth et al., 2013] & 603 & social & 9 & Sentiment & $\mathrm{P}$ & one sentiment category for each image \\
\hline FlickrCC & [Borth et al., 2013] & $\approx 500,000$ & social & - & Plutchik & $\mathrm{P}$ & one emotion category for each image \\
\hline Flickr & [Yang et al., 2014] & 301,903 & social & 6,735 & Ekman & $\mathrm{P}$ & one emotion category for each image \\
\hline Emotion6 & [Peng et al., 2015] & 1,980 & social & 432 & Ekman+neutral & $\mathrm{P}$ & the discrete probability distribution \\
\hline FI & [You et al., 2016b] & 23,308 & social & 225 & Mikels & $\mathrm{P}$ & one DEC for each image \\
\hline IESN & [Zhao et al., 2016] & $1,012,901$ & social & 118,035 & Mikels, VAD & $\mathrm{B}$ & the emotion of involved users for each image \\
\hline FlickrLDL & [Yang et al., 2017b] & 10,700 & social & 11 & Mikels & $\mathrm{P}$ & the discrete probability distribution \\
\hline TwitterLDL & [Yang et al., 2017b] & 10,045 & social & 8 & Mikels & $\mathrm{P}$ & the discrete probability distribution \\
\hline
\end{tabular}

Table 2: Released and freely available datasets for AICA, where '\# Images' and '\# Annotators' respectively represent the total number of images and annotators (f: female, m: male), 'E, P, B' are short for expected emotion, perceived emotion and both, respectively.

to 43 , STD $=5.9$ ). Besides, these images are also rated with valence and arousal values, ranging from 0 to 100 points.

The MART dataset is a collection of 500 abstract paintings from the Museum of Modern and Contemporary Art of Trento and Rovereto [Alameda-Pineda et al., 2016], which were realized by professional artists. The devArt dataset is a collection of 500 amateur abstract paintings obtained from the "DeviantArt" online social network [Alameda-Pineda et al., 2016], one of the largest online art communities. Using the relative score method in [Sartori et al., 2015], these abstract paintings are labeled as positive or negative sentiment.

Tweet dataset (Tweet) includes 470 positive tweets and 133 negative tweets [Borth et al., 2013].

The FlickrCC dataset is constructed by retrieving the Flickr creative common (CC) images for the 3,000 adjective noun pairs (ANPs) [Borth et al., 2013]. By excluding the images that do not contain the ANP string in the title, tag or description, about 500k Flickr CC images in total are generated for 1,553 ANPs. These images are then mapped to the Plutchnik's Wheel of Emotions with 8 basic emotions, each with 3 scales, such as ecstasy $\rightarrow$ joy $\rightarrow$ serenity.

The Flickr dataset consists of 301,903 images based on the Ekman emotion model [Yang et al., 2014]. A word list for each of the six emotion categories is manually defined based on WordNet and HowNet. The emotion category whose word list has the most same words as the adjective words of an image's tags and comments is assigned to the image.

The original FI dataset consists of 90,000 noisy images collected from Flicker and Instagram by searching the emotion keywords [You et al., 2016b]. The weakly labeled images are further labeled by 225 Amazon Mechanical Turk (AMT) workers, which are selected through a qualification test. The 23,308 images that receive at least three votes from their assigned 5 AMT workers are kept. The number of images in each Mikels emotion category is larger than 1,000.

The Emotion6 dataset [Peng et al., 2015] consists of 1,980 images collected from Flickr by using the emotion keywords and synonyms as search terms. There are 330 images for each emotion category. AMT workers were invited to label the images into the Ekman's 6 emotions and neutral to obtain the emotional responses. Each image was scored by 15 subjects. The discrete emotion distribution information is released.

The IESN dataset is constructed for personalized emotion prediction [Zhao et al., 2016], with 1,012,901 images from Flickr. Lexicon-based methods are used to segment the text of metadata from uploaders for expected emotions and comments from viewers for actual emotions. Synonym based searching is employed to obtain the Mikels' emotion category by selecting the most frequent synonyms. The average VAD values of the segmentation results are computed as DES ground truth based on the VAD norms of 13,915 English lemmas [Warriner et al., 2013]. 7,723 active users with more than 50 involved images are selected. The DEC and emotion distributions can also be easily obtained.

Two image datasets for discrete emotion distribution learning are released in [Yang et al., 2017b]. One is FlickrLDL dataset, a subset of FlickrCC. FlickrLDL contains 10,700 images, which are labeled by 11 viewers using Mikels' emotion model. 30,000 images are collected by searching various sentiment key words from Twitter. After duplication removal, the images are labeled by 8 viewers. In this way, the TwitterLDL dataset is generated with 10,045 images. In both datasets, the ground truth emotion distribution for each image is obtained by integrating the votes from the workers.

\section{Emotion Feature Extraction}

As shown in [Zhao et al., 2014c], there are various types of features that may contribute to the perception of image emotions. In this section, we introduce the hand-crafted features of different levels (Table 3) and the learning based features (Table 5) that have been widely extracted for AICA.

\subsection{Low-level Features}

Low-level features suffer from being difficult to be interpreted by humans. The widely used features include GIST, HOG2x2, self-similarity and geometric context color histogram features as in [Patterson and Hays, 2012], because they are each individually powerful and can describe distinct visual phenomena in a scene perspective.

Features derived from elements of art, including color and texture are extracted [Machajdik and Hanbury, 2010]. Lee and Park [2011] used the MPEG-7 visual descriptors, including four color-related ideas and two texture-related ideas. $\mathrm{Lu}$ et al. [2012] investigated how shape features in natural images influence emotions by modeling the concepts of 
Proceedings of the Twenty-Seventh International Joint Conference on Artificial Intelligence (IJCAI-18)

\begin{tabular}{|c|c|c|c|c|}
\hline Feature & Reference & Level & Short description & \# Feat \\
\hline LOW_C & [Patterson and Hays, 2012] & low & GIST, HOG2x2, self-similarity and geometric context color histogram features & 17,032 \\
\hline Elements & [Machajdik and Hanbury, 2010] & low & $\begin{array}{l}\text { color: mean saturation, brightness and hue, emotional coordinates, colorfulness, color names, Itten contrast, Wang's } \\
\text { semantic descriptions of colors, area statistics; texture: Tamura, Wavelet and gray-level co-occurrence matrix }\end{array}$ & 97 \\
\hline MPEG-7 & [Lee and Park, 2011] & low & color: layout, structure, scalable color, dominant color; texture: edge histogram, texture browsing & $\approx 200$ \\
\hline Shape & [Lu et al., 2012] & low & line segments, continuous lines, angles, curves & 219 \\
\hline IttenColor & [Sartori et al., 2015] & low & color co-occurrence features and patch-based color-combination features & 16,485 \\
\hline Attributes & [Patterson and Hays, 2012] & mid & scene attributes & 102 \\
\hline Sentributes & [Yuan et al., 2013] & mid & scene attributes, eigenfaces & 109 \\
\hline Composition & [Machajdik and Hanbury, 2010] & mid & level of detail, low depth of field, dynamics, rule of thirds & 45 \\
\hline Aesthetics & [Wang et al., 2013] & mid & figure-ground relationship, color pattern, shape, composition & 13 \\
\hline Principles & [Zhao et al., 2014a] & mid & principles-of-art: balance, contrast, harmony, variety, gradation, movement & 165 \\
\hline BoVW & [Rao et al., 2016a] & mid & bag-of-visual-words on SIFT, latent topics & 330 \\
\hline FS & [Machajdik and Hanbury, 2010] & high & number of faces and skin pixels, size of the biggest face, amount of skin w.r.t. the size of faces & 4 \\
\hline ANP & [Borth et al., 2013] & high & semantic concepts based on adjective noun pairs & 1,200 \\
\hline Expressions & [Yang et al., 2010] & high & automatically assessed facial expressions (anger, contempt, disgust, fear, happiness, sadness, surprise, neutral) & 8 \\
\hline
\end{tabular}

Table 3: Summary of the hand-crafted emotion features at different levels. '\# Feat' indicates the dimension of each feature.

\begin{tabular}{|c|c|c|c|c|c|c|}
\hline Reference & Feature & Fusion & Learning model & Dataset & Target & Result \\
\hline [Machajdik and Hanbury, 2010] & Elements, Composition, FS & early & Naive Bayes & IAPSa, Abstract, ArtPhoto & cla & $0.471,0.357,0.495$ \\
\hline [Lee and Park, 2011] & MPEG-7 & - & $K$ nearest neighbor & unreleased & cla & 0.827 \\
\hline [Lu et al., 2012] & Shape, Elements & early & SVM, SVR & IAPSa, IAPS & cla, reg & $0.314 ; \mathrm{V}-1.350, \mathrm{~A}-0.912$ \\
\hline [Li et al., 2012] & Segmented objects & - & bilayer sparse learning & IAPS, ArtPhoto & cla & $0.612,0.610$ \\
\hline [Yuan et al., 2013] & Sentributes & - & SVM, logistic regression & Tweet & cla & 0.824 \\
\hline [Wang et al., 2013] & Aesthetics & - & Naive Bayes & Abstract, ArtPhoto & cla & $0.726,0.631$ \\
\hline [Zhao et al., 2014a] & Principles & - & SVM, SVR & $\begin{array}{l}\text { IAPSa, Abstract, ArtPhoto, } \\
\text { IAPS }\end{array}$ & cla, reg & $\begin{array}{r}0.635,0.605,0.669 ; \\
\text { V-1.270, A- } 0.820\end{array}$ \\
\hline [Zhao et al., 2014c] & $\begin{array}{l}\text { LOW_C, Elements, Attributes, } \\
\text { Principles, ANP, Expressions }\end{array}$ & graph & multi-graph learning & $\begin{array}{l}\text { IAPSa, Abstract, ArtPhoto, } \\
\text { GAPED, Tweet }\end{array}$ & ret & $\begin{array}{r}0.773,0.735,0.658, \\
0.811,0.701\end{array}$ \\
\hline [Sartori et al., 2015] & IttenColor & - & sparse group Lasso & MART, devArt & cla & $0.751,0.745$ \\
\hline [Rao et al., 2016a] & BoVW & - & multiple instance learning & IAPSa, Abstract, ArtPhoto & cla & $0.699,0.636,0.707$ \\
\hline [Alameda-Pineda et al., 2016] & IttenColor & - & matrix completion & MART, devArt & cla & $0.728,0.761$ \\
\hline
\end{tabular}

Table 4: Representative DEC works on AICA using hand-crafted features, where 'Fusion' indicates the fusion strategy of different features, 'cla, reg, ret' in the Target column are short for classification, regression and retrieval (the same below), respectively, 'Result' is the reported best accuracy for classification, mean squared error for regression, and discounted cumulative gain for retrieval on the corresponding datasets.

roundness-angularity and simplicity-complexity. Based on Itten's color wheel, Sartori et al. [2015] designed two types of features to represent the color combinations.

\subsection{Mid-level Features}

Mid-level features are more interpretable, more semantic and more relevant to emotions than low-level features. 102 attributes are detected [Patterson and Hays, 2012], including 5 different types: materials, surface properties, functions or affordances, spatial envelop attributes and object presence. Besides the 102 attributes, Yuan et al. [2013] also incorporated eigenfaces corresponding to different emotions, which contribute a lot to the images containing faces. Rao et al. [2016a] extracted SIFT features as basic feature and adopted bagof-visual-words (BoVW) to represent the multi-scale blocks. The latent topic distribution estimated by probabilistic latent semantic analysis is used as another mid-level representation.

Harmonious composition is essential in an artwork and Machajdik and Hanbury [2010] extracted several features to analyze an image's compositional character. Interpretable aesthetic features [Wang et al., 2013] are designed based on the fact that artists often jointly use figure-ground relationships, color patterns, shapes and their diverse combinations to express emotions in their art creations. Features inspired from principles of art are designed in [Zhao et al., 2014a].

\subsection{High-level Features}

High-level features are the semantic contents contained in images. People can easily understand the emotions conveyed in images by recognizing the semantics. In the early years, Machajdik and Hanbury [2010] extracted simple semantic content by detecting faces and skins contained in an image. Facial expressions may determine the emotion of the images containing faces. 8 kinds of facial expressions are extracted as high-level features [Yang et al., 2010]. The expressions of images detected without faces are set as neutral. An 8 dimensional vector, each element of which indicates the number of related facial expressions in the image, is generated.

Borth et al. [2013] proposed to describe the semantic concepts by 1,200 adjective noun pairs (ANPs), which are detected by SentiBank. The advantages of ANP are that it turns a neutral noun into an ANP with strong emotions and makes the concepts more detectable, as compared to nouns and adjectives, respectively. A 1,200 dimensional double vector representing the probability of the ANPs is obtained.

Some representative works based on hand-crafted features of the above 3 levels are summarized in Table 4. Generally, high-level features (such as ANP) perform better for images with rich semantics, mid-level features (such as Principles) perform better for artistic photos, while low-level features (such as Elements) are effective for abstract paintings.

\subsection{Learning-based Features}

With the advent of deep learning, emphasis has been shifted from designing hand-crafted features to learning features in an end-to-end fashion. To tackle the weakly labeled images, You et al. [2015] proposed to progressively select a potentially cleaner subset of the training instances. An initial 
Proceedings of the Twenty-Seventh International Joint Conference on Artificial Intelligence (IJCAI-18)

\begin{tabular}{|c|c|c|c|c|c|c|c|c|}
\hline Reference & Base net & Pre & \# Feat & Cla & Loss & Dataset & Target & Result \\
\hline [You et al., 2015] & self-defined & no & 24 & - & - & FlickrCC, (unreleased) Twitter & cla & 0.781 \\
\hline [You et al., 2016b] & AlexNet & yes & 4096 & SVM & - & FI, IAPSa, Abstract, ArtPhoto & cla & $0.583,0.872,0.776,0.737$ \\
\hline [Rao et al., 2016b] & AlexNet,ACNN,TCNN & yes & $4096,256,4096$ & MIL & - & FI, IAPSa, Abstract, ArtPhoto, MART & cla & $0.652,0.889,0.825,0.834,0.764$ \\
\hline [Zhu et al., 2017] & self-defined & no & 512 & - & contrastive & FI, IAPSa, ArtPhoto & cla & $0.730,0.902,0.855$ \\
\hline [Yang et al., 2018] & GoogleNet-Inception & yes & 1024 & - & sentiment & FI, IAPSa, Abstract, ArtPhoto & cla, ret & $\begin{array}{l}0.676,0.442,0.382,0.400 \\
0.780,0.819,0.788,0.704\end{array}$ \\
\hline
\end{tabular}

Table 5: Representative works on deep learning based AICA methods, where 'Pre' indicates whether the network is pre-trained using ImageNet, '\# Feat' indicates the dimension of last feature mapping layer before the emotion output layer, 'Cla' indicates the classifier used after the last feature mapping with default Softmax, 'Loss' indicates the loss objectives besides the common cross-entropy loss, and 'Result' is the reported best accuracy for classification and discounted cumulative gain for retrieval on the corresponding datasets.

convolutional neural network $(\mathrm{CNN})$ model is trained on the training data. According to the prediction score of the trained model on the training data itself, the training samples with distinct sentiment scores between the two classes with a high probability are selected. The trained model is fine-tuned using the newly selected instances. Later, they fine-tuned the pre-trained AlexNet on ImageNet to classify emotions into 8 categories by changing the last layer of the neural network from 1000 to 8 [You et al., 2016b]. An SVM classifier is also trained using features extracted from the second to the last layer of the pre-trained AlexNet model.

Rao et al. [2016b] proposed to learn multi-level deep representations for image emotion classification (MldrNet). The input image is segmented into 3 levels of patches, which are input to 3 different CNN models, including Alexnet, aesthetics CNN (ACNN) and texture CNN (TCNN). Multiple instance learning (MIL) is employed to generate the emotion label of an input image. Based on MldrNet, Zhu et al. [2017] integrated the different levels of features by a Bidirectional GRU model (BiGRU) to exploit their dependencies. Two features generated from our Bi-GRU model are concatenated as the final features to predict the emotions. Apart from the traditional cross-entropy loss, an additional contrastive loss is jointly optimized to enforce the feature vectors extracted from each pair of images from the same category to be close enough, and those from different categories to be far away.

To explore the correlation of emotional labels with the same polarity, Yang et al. [2018] employed deep metric learning and proposed a multi-task deep framework to optimize both retrieval and classification tasks. Besides the crossentropy loss, a novel sentiment constraint is jointly optimized by considering the relations among emotional categories in the Mikels' wheel, which extends triplet constraints to a hierarchical structure. A sentiment vector based on the texture information from the convolutional layer is proposed to measure the difference between affective images.

The deep representations generally outperform the handcrafted features, which are designed based on several smallscale datasets for specific domains. However, how the deep features correlate to specific emotions is unclear.

\section{Personalized Emotion Prediction}

Zhao et al. [2016; 2018d] proposed to predict the personalized emotions (see Figure 1 (d)) of a specified user after viewing an image, associated with online social networks. Different types of factors that may influence the emotion perception are considered: the images' visual content, the so- cial context related to the corresponding users, the emotions' temporal evolution, and the images' location information. Rolling multi-task hypergraph learning is presented to jointly combine these factors. Each hypergraph vertex is a compound triple $(u, x, S)$, where $u$ represents user, $x$ and $S$ are the current image and the recent past images, termed as 'target image' and 'history image set', respectively. Based on the 3 vertex components, different types of hyperedges are constructed, including target image centric, history image set centric, and user centric hyperedges. Visual features (512dimensional GIST, Elements, Attributes, Principles, ANP, Expressions) in both target image and history image set are extracted to represent visual content. User relationship is exploited from the user component to take social context into account. Past emotion is inferred from history image set to reveal temporal evolution. Location is embedded in both target image and history image set. Semi-supervised learning is conducted on the multi-task hypergraphs to classify personalized emotions for multiple users simultaneously. The average F1 of emotion classification on the IESN dataset is 0.582 .

\section{Emotion Distribution Learning}

According to probability theory, there are typically two types of probability distributions: discrete and continuous. Generally, distribution learning can be formalized as a regression problem. For CES, the task aims to predict the discrete probability of different emotion categories, the sum of which is equal to 1 (see Figure 2). For DES, the task usually transfers to predict the parameters of specified continuous probability distributions, the form of which should be firstly determined, such as Gaussian distribution (see Figure 1 (e)) and exponential distribution.

\subsection{Discrete Probability Distribution Learning}

Zhao et al. [2015] proposed shared sparse learning (SSL) to represent the probability distribution of one test image as the linear combination of the training images' distributions, the coefficients of which are learned from the feature space. Only uni-modal features are considered in SSL, which can simply adopt early or late fusion to handle multi-modal features (indicate multiple features from images unless otherwise specified) without considering the latent correlations. Multi-modal features are fused by weighted multi-modal SSL (WMMSSL) [Zhao et al., 2017a; 2018a] to explore useful information by the constraint of joint sparsity across different features. The representation abilities of different features are jointly explored with the optimal weight automatically learned. 
Proceedings of the Twenty-Seventh International Joint Conference on Artificial Intelligence (IJCAI-18)

\begin{tabular}{|c|c|c|c|c|c|c|}
\hline Reference & Feature & & Fusion & Learning model & Dataset & Result \\
\hline [Zhao et al., 2015] & \multicolumn{2}{|c|}{ GIST, Elements, Principles } & - & SSL & Abstract & 0.134 \\
\hline [Zhao et al., 2017a] & \multicolumn{2}{|c|}{$\begin{array}{l}\text { GIST, Elements, Attributes, Principles, } \\
\text { ANP, deep features from AlexNet }\end{array}$} & weighted & WMMSSL & Abstract, Emotion6, IESN & $0.482,0.479,0.478$ \\
\hline [Yang $e$ & \multirow{2}{*}{\multicolumn{2}{|c|}{$\begin{array}{l}\text { ANP, deep features from VGG16 } \\
\text { GIST, Elements, Attributes, Principles, } \\
\text { ANP, deep features from AlexNet }\end{array}$}} & - & augmented CPNN & Abstract, Emotion6, FlickrLDL, TwitterLDL & $0.480,0,506,0,469,0.555$ \\
\hline [Zhao et al., 2017b] & & & weighted & WMMCPNN & Abstract, Emotion6, IESN & $0.461,0.464,0.470$ \\
\hline Reference & Base net & Pre & \# Output & Loss & Dataset & Result \\
\hline [Peng et al., 2015] & AlexNet & yes & 1 & Euclidean loss & Emotion6 & 0.480 \\
\hline [Yang et al., 2017a] & VGG16 & yes & 6 or 8 & KL divergence loss & Emotion6, FlickrLDL, TwitterLDL & $0.420,0,530,0,530$ \\
\hline
\end{tabular}

Table 6: Representative discrete distribution learning works on AICA, where 'Fusion' indicates the fusion strategy of different features, 'Pre' indicates whether the network is pre-trained using ImageNet, '\# Output' is the output dimension of the last layer, and 'Result' is the reported best KL divergence on the corresponding datasets except the first line, which is the result on sum of squared difference.

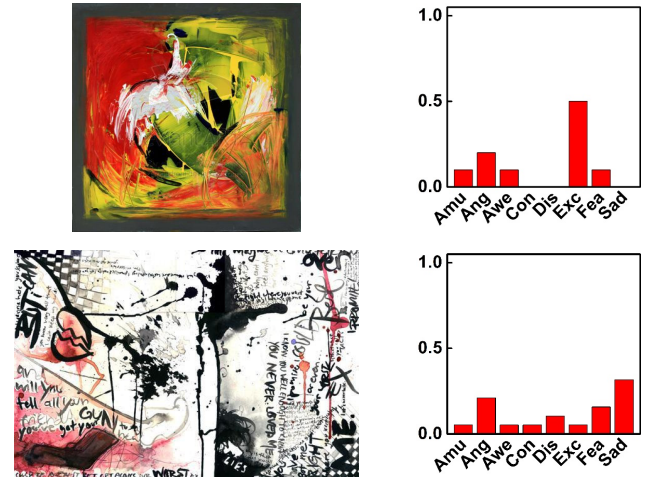

Figure 2: Examples of the image emotion's discrete probability distribution [Zhao et al., 2018a].

Both SSL and WMMSSL can only model one test image each time. For different test images, the shared coefficients have to be learned repeatedly. Geng et al. [2013] proposed a conditional probability neural network (CPNN) for distribution learning by modeling the conditional probability of labels given features as a three layer neural network. Yang et al. [2017b] replaced the signless integers in CPNN with a binary representation, since adding two emotion labels or subtracting one label from another is meaningless. By adding noises to the ground truth emotion labels, more roughly labeled distributions and samples are generated. Zhao et al. [2017b] extended CPNN into multi-modal settings and proposed weighted multi-model CPNN (WMMCPNN) to jointly explore the representation abilities of different features. The linear combination of different CPNN loss functions are minimized with a sparse constraint on the combination coefficients. Once the parameters of the augmented CPNN and WMMCPNN are learned, the emotion distributions of a test image would be easily obtained.

The CPNN series have only 3 layers and the input is extracted visual features instead of original images. A deeper $\mathrm{CNN}$ regressor (CNNR) for each emotion category is trained in Emotion6 [Peng et al., 2015] with the exact AlexNet architecture. The number of output nodes is changed to 1 to predict a real value and the Softmax loss layer is replaced with the Euclidean loss layer. The predicted probabilities of all emotion categories are normalized to sum to 1. However, the CNNR cannot guarantee that the predicted probability is non-negative. Further, the probability correlations among different emotions are actually ignored, since the regressor for each emotion category is trained independently. Yang et al. [2017a] designed a multi-task deep framework based on VGG16 by jointly optimizing the cross-entropy loss for emotion classification and Kullback-Leibler (KL) divergence loss for emotion distribution learning, which achieves the stateof-the-art performances. For the single emotion dataset, the single label is transformed to emotion distribution with emotion distances computed on Mikels' wheel. The representative methods are summarize in Table 6.

\subsection{Continuous Probability Distribution Learning}

Zhao et al. [2017c] proposed to learn continuous emotion distributions in VA space. Based on the assumption that the VA emotion labels can be well modeled by a mixture of 2 bidimensional Gaussian mixture models (GMMs, see Figure 1 (e)), the task turns to predict the parameters of GMMs, including the mean vector and covariance matrix of the 2 Gaussian components as well as the mixing coefficients.

Shared sparse regression (SSR) is proposed as the learning model by assuming that the test feature and test parameters can be linearly represented by the training features and training parameters but with shared coefficients. SSR can only model one test image each time. To explore the task relatedness, multi-task SSR is further presented to simultaneously predict the parameters of different test images by utilizing appropriate shared information across tasks. GIST, Elements, Attributes, Principles, ANP, and 4,096-dimensional deep features from AlexNet are extracted as visual features. Experiments are conducted on a subset of IESN, which consists of 18,700 images each with more than 20 VA labels. The average KL divergence of multi-task SSR using ANP is 0.436 .

\section{Conclusion and Future Directions}

This paper attempted to provide an overview of recent developments on affective image content analysis (AICA). Obviously, it cannot cover all the literature on AICA, and we focused on a representative subset of the latest methods. We summarized the widely employed emotion representation models, released datasets, and compared the existing methods on emotion feature extraction, personalized emotion prediction and emotion distribution learning. We believe that AICA will continue to be an active and promising research area with broad potential applications, such as an emotion-aware personalized music slide show [Chen et al., 2014], emotion based image musicalization [Zhao et al., 2014b], and image 
captioning with sentiment [Mathews et al., 2016]. Many issues in AICA, however, are still open.

Understanding image content and context. Accurately analyzing what is contained in an image can significantly improve the performance of AICA. Sometimes we even need subtle analysis of visual contents. For example, if an image is about the laugh of a lovely child, it is more likely that we feel "amused"; but if it is about the laugh of a known evil ruler or criminal, we may feel "angry". The context of image content is also important in AICA. Similar visual content under different contexts may evoke different emotions. For example, we may feel "happy" about beautiful flowers. But if the flowers are placed in a funeral, we possibly feel "sad".

Recognizing group emotions. Recognizing the dominant emotion is too generic, while predicting personalized emotion is too specific. Modeling emotions for groups or cliques of users, who share similar tastes or interests, may be a good choice - e.g., by cultural or societal background. Analyzing the user profiles provided by each individual to classify users into different types of backgrounds, tastes and interests may help to tackle this problem.

Understanding emotions of 3D data and videos. Compared with traditional intensity images, 3D data contain more spatial information, being useful in low light levels and being color and texture invariant, while videos (such as animated GIF) contain rich temporal correlation information. Combining the spatial and temporal correlation together with the visual content would make more sense.

Further, jointly exploring the complementarity of multimodal data (such as image and text) [You et al., 2016a; Zhao et al., 2018c; Chen et al., 2018] would improve the emotion recognition performance. How to adapt the emotions from one labeled domain to another unlabeled domain [Patel et al., 2015] is another interesting research topic.

\section{Acknowledgments}

This work is supported by the Berkeley Deep Drive, the National Natural Science Foundation of China (Nos. 61701273, 61571269, 61332016, 61620106009, U1636214), the China Postdoctoral Science Foundation Project (No. 2017M610897), and the European Union's Horizon 2020 Programme through the Innovative Action No. 645094 (SEWA). This research is part of NExT++ project, supported by the National Research Foundation, Prime Ministers Office, Singapore under its IRC@Singapore Funding Initiative.

\section{References}

[Alameda-Pineda et al., 2016] Xavier Alameda-Pineda, Elisa Ricci, Yan Yan, and Nicu Sebe. Recognizing emotions from abstract paintings using non-linear matrix completion. In IEEE Conference on Computer Vision and Pattern Recognition, pages 52405248, 2016.

[Borth et al., 2013] Damian Borth, Rongrong Ji, Tao Chen, Thomas Breuel, and Shih-Fu Chang. Large-scale visual sentiment ontology and detectors using adjective noun pairs. In ACM International Conference on Multimedia, pages 223-232, 2013.

[Chen et al., 2014] Tao Chen, Felix X Yu, Jiawei Chen, Yin Cui, Yan-Ying Chen, and Shih-Fu Chang. Object-based visual sen- timent concept analysis and application. In ACM International Conference on Multimedia, pages 367-376, 2014.

[Chen et al., 2018] Fuhai Chen, Rongrong Ji, Jinsong Su, Donglin $\mathrm{Cao}$, and Yue Gao. Predicting microblog sentiments via weakly supervised multimodal deep learning. IEEE Transactions on Multimedia, 20(4):997-1007, 2018.

[Dan-Glauser and Scherer, 2011] Elise S Dan-Glauser and Klaus R Scherer. The geneva affective picture database (gaped): a new 730-picture database focusing on valence and normative significance. Behavior Research Methods, 43(2):468-477, 2011.

[Ekman, 1992] Paul Ekman. An argument for basic emotions. Cognition \& Emotion, 6(3-4):169-200, 1992.

[Geng et al., 2013] Xin Geng, Chao Yin, and Zhi-Hua Zhou. Facial age estimation by learning from label distributions. IEEE Transactions on Pattern Analysis and Machine Intelligence, 35(10):2401-2412, 2013.

[Hanjalic, 2006] Alan Hanjalic. Extracting moods from pictures and sounds: Towards truly personalized tv. IEEE Signal Processing Magazine, 23(2):90-100, 2006.

[Lang et al., 1997] Peter J Lang, Margaret M Bradley, and Bruce N Cuthbert. International affective picture system (iaps): Technical manual and affective ratings. NIMH Center for the Study of Emotion and Attention, pages 39-58, 1997.

[Lee and Park, 2011] Joonwhoan Lee and EunJong Park. Fuzzy similarity-based emotional classification of color images. IEEE Transactions on Multimedia, 13(5):1031-1039, 2011.

[Li et al., 2012] Bing Li, Weihua Xiong, Weiming Hu, and Xinmiao Ding. Context-aware affective images classification based on bilayer sparse representation. In ACM International Conference on Multimedia, pages 721-724, 2012.

[Lu et al., 2012] Xin Lu, Poonam Suryanarayan, Reginald B Adams Jr, Jia Li, Michelle G Newman, and James Z Wang. On shape and the computability of emotions. In ACM International Conference on Multimedia, pages 229-238, 2012.

[Machajdik and Hanbury, 2010] Jana Machajdik and Allan Hanbury. Affective image classification using features inspired by psychology and art theory. In ACM International Conference on Multimedia, pages 83-92, 2010.

[Mathews et al., 2016] Alexander Patrick Mathews, Lexing Xie, and Xuming He. Senticap: Generating image descriptions with sentiments. In AAAI Conference on Artificial Intelligence, pages 3574-3580, 2016.

[Mikels et al., 2005] Joseph A Mikels, Barbara L Fredrickson, Gregory R Larkin, Casey M Lindberg, Sam J Maglio, and Patricia A Reuter-Lorenz. Emotional category data on images from the international affective picture system. Behavior Research Methods, 37(4):626-630, 2005.

[Patel et al., 2015] Vishal M Patel, Raghuraman Gopalan, Ruonan $\mathrm{Li}$, and Rama Chellappa. Visual domain adaptation: A survey of recent advances. IEEE Signal Processing Magazine, 32(3):5369, 2015.

[Patterson and Hays, 2012] Genevieve Patterson and James Hays. Sun attribute database: Discovering, annotating, and recognizing scene attributes. In IEEE Conference on Computer Vision and Pattern Recognition, pages 2751-2758, 2012.

[Peng et al., 2015] Kuan-Chuan Peng, Amir Sadovnik, Andrew Gallagher, and Tsuhan Chen. A mixed bag of emotions: Model, predict, and transfer emotion distributions. In IEEE Conference on Computer Vision and Pattern Recognition, pages 860$868,2015$. 
[Plutchik, 1980] Robert Plutchik. Emotion: A psychoevolutionary synthesis. Harpercollins College Division, 1980.

[Rao et al., 2016a] Tianrong Rao, Min Xu, Huiying Liu, Jinqiao Wang, and Ian Burnett. Multi-scale blocks based image emotion classification using multiple instance learning. In IEEE International Conference on Image Processing, pages 634-638, 2016.

[Rao et al., 2016b] Tianrong Rao, Min Xu, and Dong Xu. Learning multi-level deep representations for image emotion classification. arXiv preprint arXiv:1611.07145, 2016.

[Sartori et al., 2015] Andreza Sartori, Dubravko Culibrk, Yan Yan, and Nicu Sebe. Who's afraid of itten: Using the art theory of color combination to analyze emotions in abstract paintings. In ACM International Conference on Multimedia, pages 311-320, 2015.

[Schlosberg, 1954] Harold Schlosberg. Three dimensions of emotion. Psychological Review, 61(2):81, 1954.

[Sun et al., 2009] Kai Sun, Junqing Yu, Yue Huang, and Xiaoqiang Hu. An improved valence-arousal emotion space for video affective content representation and recognition. In IEEE International Conference on Multimedia and Expo, pages 566-569, 2009.

[Wang et al., 2013] Xiaohui Wang, Jia Jia, Jiaming Yin, and Lianhong Cai. Interpretable aesthetic features for affective image classification. In IEEE International Conference on Image Processing, pages 3230-3234, 2013.

[Warriner et al., 2013] Amy Beth Warriner, Victor Kuperman, and Marc Brysbaert. Norms of valence, arousal, and dominance for 13,915 english lemmas. Behavior Research Methods, 45(4):1191-1207, 2013.

[Yang et al., 2010] Peng Yang, Qingshan Liu, and Dimitris N Metaxas. Exploring facial expressions with compositional features. In IEEE Conference on Computer Vision and Pattern Recognition, pages 2638-2644, 2010.

[Yang et al., 2014] Yang Yang, Jia Jia, Shumei Zhang, Boya Wu, Qicong Chen, Juanzi Li, Chunxiao Xing, and Jie Tang. How do your friends on social media disclose your emotions? In $A A A I$ Conference on Artificial Intelligence, pages 306-312, 2014.

[Yang et al., 2017a] Jufeng Yang, Dongyu She, and Ming Sun. Joint image emotion classification and distribution learning via deep convolutional neural network. In International Joint Conference on Artificial Intelligence, pages 3266-3272, 2017.

[Yang et al., 2017b] Jufeng Yang, Ming Sun, and Xiaoxiao Sun. Learning visual sentiment distributions via augmented conditional probability neural network. In AAAI Conference on Artificial Intelligence, pages 224-230, 2017.

[Yang et al., 2018] Jufeng Yang, Dongyu She, Yukun Lai, and Ming-Hsuan Yang. Retrieving and classifying affective images via deep metric learning. In AAAI Conference on Artificial Intelligence, 2018.

[You et al., 2015] Quanzeng You, Jiebo Luo, Hailin Jin, and Jianchao Yang. Robust image sentiment analysis using progressively trained and domain transferred deep networks. In AAAI Conference on Artificial Intelligence, pages 381-388, 2015.

[You et al., 2016a] Quanzeng You, Liangliang Cao, Hailin Jin, and Jiebo Luo. Robust visual-textual sentiment analysis: When attention meets tree-structured recursive neural networks. In ACM International Conference on Multimedia, pages 1008-1017, 2016.

[You et al., 2016b] Quanzeng You, Jiebo Luo, Hailin Jin, and Jianchao Yang. Building a large scale dataset for image emotion recognition: The fine print and the benchmark. In AAAI Conference on Artificial Intelligence, pages 308-314, 2016.
[Yuan et al., 2013] Jianbo Yuan, Sean Mcdonough, Quanzeng You, and Jiebo Luo. Sentribute: image sentiment analysis from a midlevel perspective. In ACM International Workshop on Issues of Sentiment Discovery and Opinion Mining, page 10, 2013.

[Zhao et al., 2014a] Sicheng Zhao, Yue Gao, Xiaolei Jiang, Hongxun Yao, Tat-Seng Chua, and Xiaoshuai Sun. Exploring principles-of-art features for image emotion recognition. In ACM International Conference on Multimedia, pages 47-56, 2014.

[Zhao et al., 2014b] Sicheng Zhao, Hongxun Yao, Fanglin Wang, Xiaolei Jiang, and Wei Zhang. Emotion based image musicalization. In IEEE International Conference on Multimedia and Expo Workshops, pages 1-6, 2014.

[Zhao et al., 2014c] Sicheng Zhao, Hongxun Yao, You Yang, and Yanhao Zhang. Affective image retrieval via multi-graph learning. In ACM International Conference on Multimedia, pages 1025-1028, 2014.

[Zhao et al., 2015] Sicheng Zhao, Hongxun Yao, Xiaolei Jiang, and Xiaoshuai Sun. Predicting discrete probability distribution of image emotions. In IEEE International Conference on Image Processing, pages 2459-2463, 2015.

[Zhao et al., 2016] Sicheng Zhao, Hongxun Yao, Yue Gao, Rongrong Ji, Wenlong Xie, Xiaolei Jiang, and Tat-Seng Chua. Predicting personalized emotion perceptions of social images. In ACM International Conference on Multimedia, pages 13851394, 2016.

[Zhao et al., 2017a] Sicheng Zhao, Guiguang Ding, Yue Gao, and Jungong Han. Approximating discrete probability distribution of image emotions by multi-modal features fusion. In International Joint Conference on Artificial Intelligence, pages 4669-4675, 2017.

[Zhao et al., 2017b] Sicheng Zhao, Guiguang Ding, Yue Gao, and Jungong Han. Learning visual emotion distributions via multimodal features fusion. In ACM International Conference on Multimedia, pages 369-377, 2017.

[Zhao et al., 2017c] Sicheng Zhao, Hongxun Yao, Yue Gao, Rongrong Ji, and Guiguang Ding. Continuous probability distribution prediction of image emotions via multi-task shared sparse regression. IEEE Transactions on Multimedia, 19(3):632-645, 2017.

[Zhao et al., 2018a] Sicheng Zhao, Guiguang Ding, Yue Gao, Xin Zhao, Youbao Tang, Jungong Han, Hongxun Yao, and Qingming Huang. Discrete probability distribution prediction of image emotions with shared sparse learning. IEEE Transactions on Affective Computing, 2018.

[Zhao et al., 2018b] Sicheng Zhao, Guiguang Ding, Jungong Han, and Yue Gao. Personality-aware personalized emotion recognition from physiological signals. In International Joint Conference on Artificial Intelligence, 2018.

[Zhao et al., 2018c] Sicheng Zhao, Yue Gao, Guiguang Ding, and Tat-Seng Chua. Real-time multimedia social event detection in microblog. IEEE Transactions on Cybernetics, 2018.

[Zhao et al., 2018d] Sicheng Zhao, Hongxun Yao, Yue Gao, Guiguang Ding, and Tat-Seng Chua. Predicting personalized image emotion perceptions in social networks. IEEE Transactions on Affective Computing, 2018.

[Zhu et al., 2017] Xinge Zhu, Liang Li, Weigang Zhang, Tianrong Rao, Min Xu, Qingming Huang, and Dong Xu. Dependency exploitation: a unified cnn-rnn approach for visual emotion recognition. In International Joint Conference on Artificial Intelligence, pages 3595-3601, 2017. 